(6) OPEN ACCESS

\title{
Therapeutic interleukin- 6 blockade reverses transforming growth factor-beta pathway activation in dermal fibroblasts: insights from the faSscinate clinical trial in systemic sclerosis
}

\author{
Christopher P Denton, ${ }^{1}$ Voon H Ong, ${ }^{1}$ Shiwen $\mathrm{Xu}_{1}{ }^{1}$ Haiyin Chen-Harris, ${ }^{2}$ \\ Zora Modrusan, ${ }^{2}$ Robert Lafyatis, ${ }^{3}$ Dinesh Khanna, ${ }^{4}$ Angelika Jahreis, ${ }^{2}$ Jeffrey Siegel, ${ }^{2}$ \\ Thierry Sornasse ${ }^{2}$
}

\begin{abstract}
Handling editor Josef S Smolen

- Additional material is published online only. To view please visit the journal online (http://dx.doi.org/10.1136/ annrheumdis-2018-213031).
\end{abstract}

'Division of Medicine, Royal Free Campus, University College London, London, UK ${ }^{2}$ Genentech, South San Francisco, California, USA ${ }^{3}$ University of Pittsburgh Medical Center, Pittsburgh Pennsylvania, USA

${ }^{4}$ University of Michigan Scleroderma Program, Ann Arbor, Michigan, USA

Correspondence to Professor Christopher P Denton, University College London Division of Medicine, Royal Free Campus, London, NW3 2PF, UK; c.denton@ucl.ac.uk

Received 16 January 2018 Revised 23 April 2018 Accepted 25 April 2018 Published Online First 31 May 2018

\section{ABSTRACT}

Objectives Skin fibrosis mediated by activated dermal fibroblasts is a hallmark of systemic sclerosis (SSc), especially in the subset of patients with diffuse disease. Transforming growth factor-beta (TGF $\beta$ ) and interleukin-6 (IL-6) are key candidate mediators in SSC. Our aim was to elucidate the specific effect of IL- 6 pathway blockade on the biology of SSc fibroblasts in vivo by using samples from a unique clinical experiment- the faSScinate study - in which patients with SSc were treated for 24 weeks with tocilizumab (TCZ), an IL-6 receptor- $\alpha$ inhibitor.

Methods We analysed the molecular, functional and genomic characteristics of explant fibroblasts cultured from matched skin biopsy samples collected at baseline and at week 24 from 12 patients receiving placebo $(n=6)$ or TCZ $(n=6)$ and compared these with matched healthy control fibroblast strains.

Results The hallmark functional and molecularactivated phenotype was defined in SSc samples and was stable over 24 weeks in placebo-treated cases. RNA sequencing analysis robustly defined key dysregulated pathways likely to drive SSc fibroblast activation in vivo. Treatment with TCZ for 24 weeks profoundly altered the biological characteristics of explant dermal fibroblasts by normalising functional properties and reversing gene expression profiles dominated by TGF $\beta$-regulated genes and molecular pathways.

Conclusions We demonstrated the exceptional value of using explant dermal fibroblast cultures from a well-designed trial in SSc to provide a molecular framework linking IL-6 to key profibrotic pathways. The profound impact of IL-6R blockade on the activated fibroblast phenotype highlights the potential of IL- 6 as a therapeutic target in SSc and other fibrotic diseases. Trial registration number NCT01532869; Postresults.

\section{INTRODUCTION}

Systemic sclerosis (SSc) is characterised by fibrosis of the skin and internal organs, autoimmunity and vasculopathy. ${ }^{1}$ Although the biological mechanisms underlying SSc are not fully understood, interplay between vasculopathy, autoimmunity and fibrosis may perpetuate a state of aberrant wound repair. ${ }^{2}$ Disease modification remains elusive, and treatment focuses on complications such as interstitial lung disease, pulmonary artery hypertension and peripheral vasculopathy. ${ }^{3}$ Clinical heterogeneity is a hallmark of SSc, and patients with severe progressive diffuse skin involvement have poor outcomes; more effective treatment for this subset of patients is an important unmet medical need. ${ }^{4}$

Fibroblasts play a central role in the biology of the disease ${ }^{5}$ by interacting with endothelial cells $^{6}$ and leucocytes ${ }^{7}$ in a complex biological network involving cytokines and adhesion molecules ${ }^{8}$ resulting in excess deposition of extracellular matrix proteins and in tissue stiffening. Transforming growth factor-beta (TGF $\beta$ ) plays a central role in fibrosis ${ }^{9}$ by promoting the differentiation of fibroblasts into myofibroblasts and by inducing profibrotic molecules and proliferation. ${ }^{10}$ Interleukin-6 (IL-6) also plays an important role in $\mathrm{SSc}^{11}$ by regulating the function of immune and non-immune cells. Dermal fibroblasts from patients with SSc express increased levels of IL- $6^{12}$; elevated levels of IL-6 are associated with early disease, ${ }^{13}$ and increased serum IL- 6 level predicts higher mortality risk, worse skin involvement and increased pulmonary decline. ${ }^{14}{ }^{15}$ IL- 6 is known to induce TGF $\beta$ production by cardiac fibroblasts ${ }^{16}$ and to enhance TGF $\beta$-signalling in dermal fibroblasts ${ }^{17}$ and cardiac fibroblasts. ${ }^{18}$ Conversely, TGF $\beta$ regulates the expression of IL- 6 by lung fibroblasts ${ }^{19}$ and airway smooth muscle cells. ${ }^{20}$

To elucidate the specific effect of IL-6 on the biology of fibroblasts in vivo, we took advantage of a unique clinical experiment-the faSScinate study-in which SSc patients were treated for 24 weeks with tocilizumab (TCZ), an IL-6 receptor- $\alpha$ blocking antibody. The faSScinate study was a randomised, double-blind, placebo (PBO)-controlled phase 2 study of TCZ in adult SSc patients with $<5$ years' disease duration, a modified Rodnan skin score (mRSS) between 15 and 40 units and active progressive disease according to specified clinical or laboratory features. No concomitant immunosuppressive medication was permitted at study entry or during the first 24 weeks of the trial. ${ }^{21}$ In this study, 43 patients with SSc received weekly TCZ $162 \mathrm{mg}$ subcutaneously and 44 patients with SSc received weekly $\mathrm{PBO}$ subcutaneously. As previously reported, primary clinical data 
from the faSScinate trial showed a trend of benefit in favour of TCZ for the primary endpoint or mRSS and a strong trend at 48 weeks together with congruent benefit in exploratory endpoints including lung function. Based on these results, a phase 3 trial of TCZ is under way (ClinicalTrials.gov, NCT02453256).

Here we define the molecular and functional phenotype of explant dermal fibroblasts from a representative subset of patients with SSc enrolled into faSScinate and demonstrate a profound reversal of the hallmark profibrotic properties of these cells after treatment with TCZ for 24 weeks compared with controls from the PBO arm of the trial.

\section{METHODS}

\section{Sample collection and analysis}

Culture of dermal explant fibroblasts from patients with SSc enrolled in the faSScinate study (ClinicalTrials.gov, NCT01532869) and matched controls was performed as described, as was protein quantification using Western blot analysis. ${ }^{15}$ Cell migration was assessed using scratch wound assay, ${ }^{22}$ and contractility assays were performed on 3D collagen gel lattices. ${ }^{23}$ Gene expression patterns were analysed using Ingenuity Pathway Analysis (IPA; Qiagen). Additional methodology is described in online supplementary appendix S1.

\section{Statistical analysis}

For functional assays, means were compared using Tukey's multiple comparison test. Correlations were calculated using the Spearman $\rho$. The Benjamini and Hochberg False Discovery Rate method was used to correct for multiple comparisons. All statistical calculations were conducted in JMP V.11.1 (SAS Institute).

\section{RESULTS}

\section{SSc explant dermal fibroblasts present hallmark functional properties of activated fibrotic cells}

Dermal fibroblasts were cultured from fresh skin biopsy samples collected at baseline and at week 24 from consecutive PBO-treated and TCZ-treated patients enrolled in the faSScinate study (online supplementary table S1). ${ }^{21}$ Confirming earlier reports, ${ }^{22}$ dermal fibroblasts isolated from patients with SSc at baseline produced significantly higher levels of connective tissue growth factor (CTGF), collagen alpha 1 (Col1) and alpha-smooth muscle actin ( $\alpha$ SMA) than normal fibroblasts (NFs) (NF vs SSc; $<<0.0001$ for all three factors) (figure $1 \mathrm{~A}-\mathrm{C}$ ). Migratory capacity and contractility activity were also enhanced compared with NF (NF vs PBO baseline and NF vs TCZ baseline, $\mathrm{p}<0.0001$ for both) (figure $1 \mathrm{D}, \mathrm{E}$ ). These results indicate that the SSc explant dermal fibroblasts obtained in this study have the hallmark functional characteristics of activated fibrotic fibroblasts.

\section{SSc dermal fibroblasts collected at baseline: typical profibrotic signature dominated by genes regulated by TGF $\beta$}

Using RNA sequencing (RNA-Seq), we compared the expression profiles of SSc fibroblasts collected at baseline (SSc) with those of NF. Figure 2 depicts the expression values of NF and SSc baseline fibroblasts for 578 protein-coding genes significantly differentially expressed (criteria: fold difference $\geq 1.5$; median reads per kilobase of transcript per million mapped reads $\geq 1$, adjusted $\mathrm{p} \leq 0.05)$. Of the 578 selected genes, 145 are known to be regulated by TGF $\beta$ in human dermal or lung fibroblasts, ${ }^{2024-26}$ indicating that this pathway is dominant in these cells (figure 2). Of note, baseline erythrocyte sedimentation rate (ESR) and C reactive protein and IL-6 levels were not associated with distinct baseline gene expression profiles (online supplementary table S2).

\section{TCZ treatment for $\mathbf{2 4}$ weeks attenuates the functional properties of SSc explant fibroblasts}

Comparison of functional measures between fibroblasts collected at baseline and week 24 from patients treated with TCZ showed a significant decrease in protein production, in migration (increase in gap size) and in contractility (increase in gel weight) (figure 3), indicating that therapeutic treatment with TCZ results in a significant attenuation of the hallmark functional properties of SSc fibroblasts. In contrast, the phenotype of SSc fibroblasts was stable in PBO-treated patients.

\section{Profibrotic expression profile of SSc dermal fibroblasts: normalised in TCZ-treated patients and stable in PBO-treated patients}

Figure 4 depicts expression levels for the PBO and TCZ groups at week 24 compared with the matching sample at baseline. In the PBO-treated group (figure 4), no significant difference was detected (see also online supplementary figure S1), indicating that baseline expression profile is sustained over $\geq 24$ weeks in the absence of immunosuppressants. In contrast, in the TCZ group (figure 4), we identified 2136 significantly differentially expressed genes; of those, 411 were also differentially expressed in the SSc/NF comparison. Remarkably, the expression pattern of the 411 shared genes in the TCZ group was opposite to that observed in the SSc versus NF comparison (figure 4). Of note, 167 and 1725 genes were significantly differentially expressed in SSc/NF and TCZ week 24/baseline comparisons, respectively. For most genes, differential expression trends were similar to those observed in the 411 shared genes (online supplementary figure S2). Thus, gene expression data confirm that therapeutic treatment with TCZ for 24 weeks profoundly alters the SSc-associated molecular phenotype of explanted dermal fibroblasts. Considering this striking biological effect of TCZ, we explored the correlation between gene expression and mRSS across all patients and time points. Of the 411 genes analysed, 35 had a nominal correlation $\mathrm{p} \leq 0.05$ (online supplementary table S3), and only one, PRKCE, remained significant after correction for multiple testing. Treatment with TCZ resulted in a significant upregulation of PRKCE expression that was underexpressed in SSc dermal fibroblasts at baseline, as previously reported in SSc lung fibroblasts. ${ }^{27}$

\section{Treatment with TCZ for 24 weeks: reversal of activation status of key pathological pathways associated with SSc}

We used the IPA (Qiagen) to assign biological functions to networks of all significantly differentially expressed genes (2303 genes) from the SSc versus NF and the TCZ week 24 versus baseline comparisons (online supplementary figure S1). Of the 609 significantly enriched pathways, we selected the top 50 pathways with the highest combined absolute $\mathrm{Z}$ scores (figure 5) and grouped them into 10 major biological nodes; annotations for the selected pathways are provided in (online supplementary table S4). Most pathways defined by genes that were only differentially expressed in one comparison overlapped with the 50 pathways.

The fibrosis node is dominated by TGF $\beta$-related pathways but also includes a pathway related to the activity of lysophosphatidic acid and to the antifibrotic transcription factor KLF2. The contraction node regroups 11 pathways related to cellular contractility machinery. The migration node includes four pathways positively 


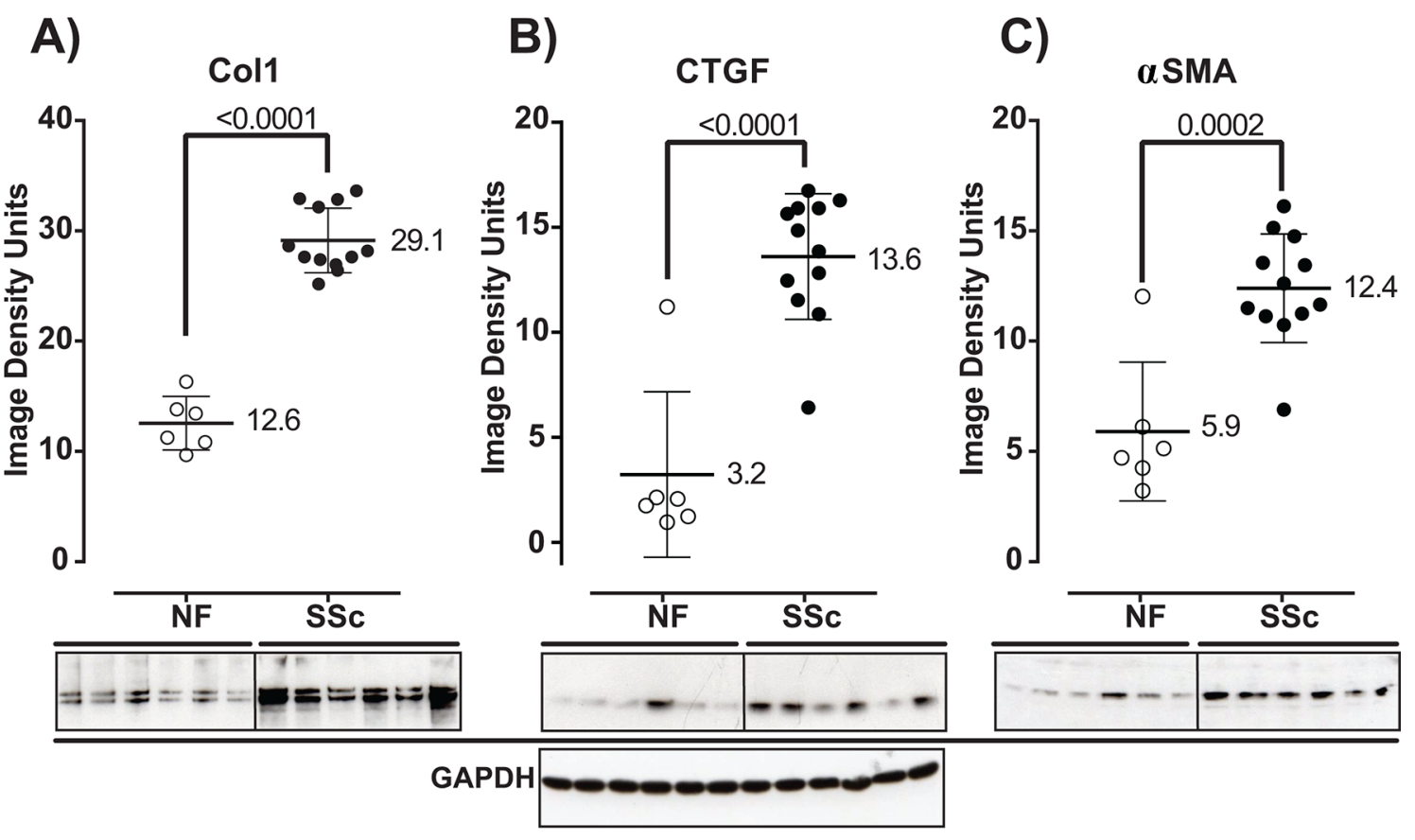

D)

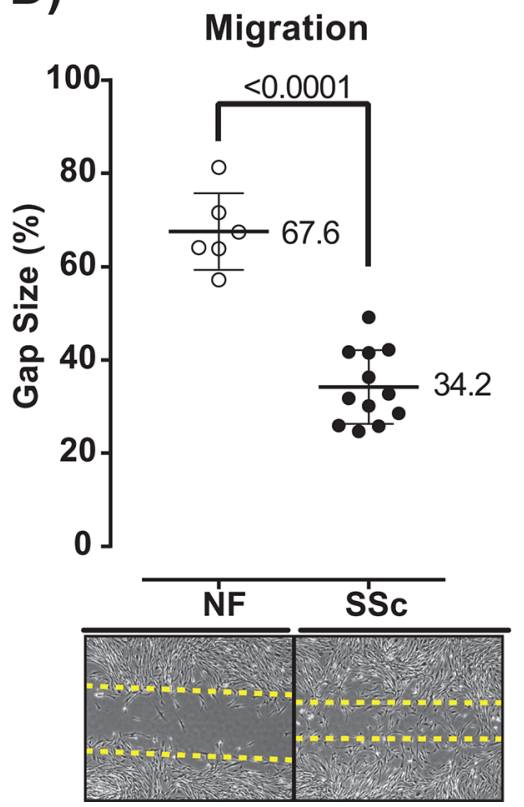

E)

\section{Contraction}
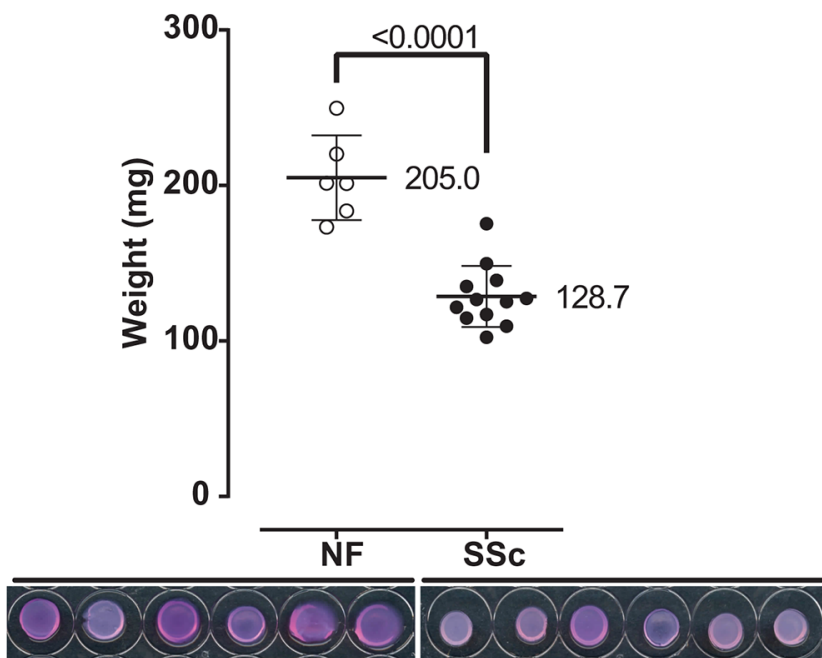

Figure 1 Explant dermal fibroblasts present hallmark functional properties of activated fibrotic fibroblasts. Production of Col1 (A), CTGF (B) and $\alpha S M A(C)$; migration activity (D); and contractility (E) by normal and SSc dermal fibroblasts collected at baseline were analysed by western blot densitometry of cell lysates $(A-C)$, scratch assay: percentage of remaining gap size $(D)$ and $3 D$ gel contraction assay: weight of the lattice plug $(E)$, respectively. Representative western blot images are presented for each protein analysed (A-C, lower panel); western blot of the reference protein glyceraldehyde 3-phosphate dehydrogenase (GAPDH) is shown for the corresponding samples. Representative images of scratch assay performed over an incubation period of 48 hours are shown ( $D$, lower panel). Representative images of gel contraction assay performed over an incubation period of 24 hours are shown (E, lower panel). BL, baseline; Col1, collagen alpha 1; CTGF, connective tissue growth factor; NFs, normal fibroblasts; $\alpha$ SMA, alpha-smooth muscle actin; SSc, systemic sclerosis.

related to cell movement. The growth node contains three pathways positively related to proliferation and cell survival. The five pathways grouped under the cardiovascular node are generally associated with endothelial and vascular smooth muscle cell biology but are also known to be positively related to fibrosis.
Although the main function of the two pathways grouped under the coagulation node is blood clotting, these pathways play an important role in linking vascular events to fibrotic events in SSc. The activation node regroups eight pathways representing major signalling molecules and transcription factors positively 


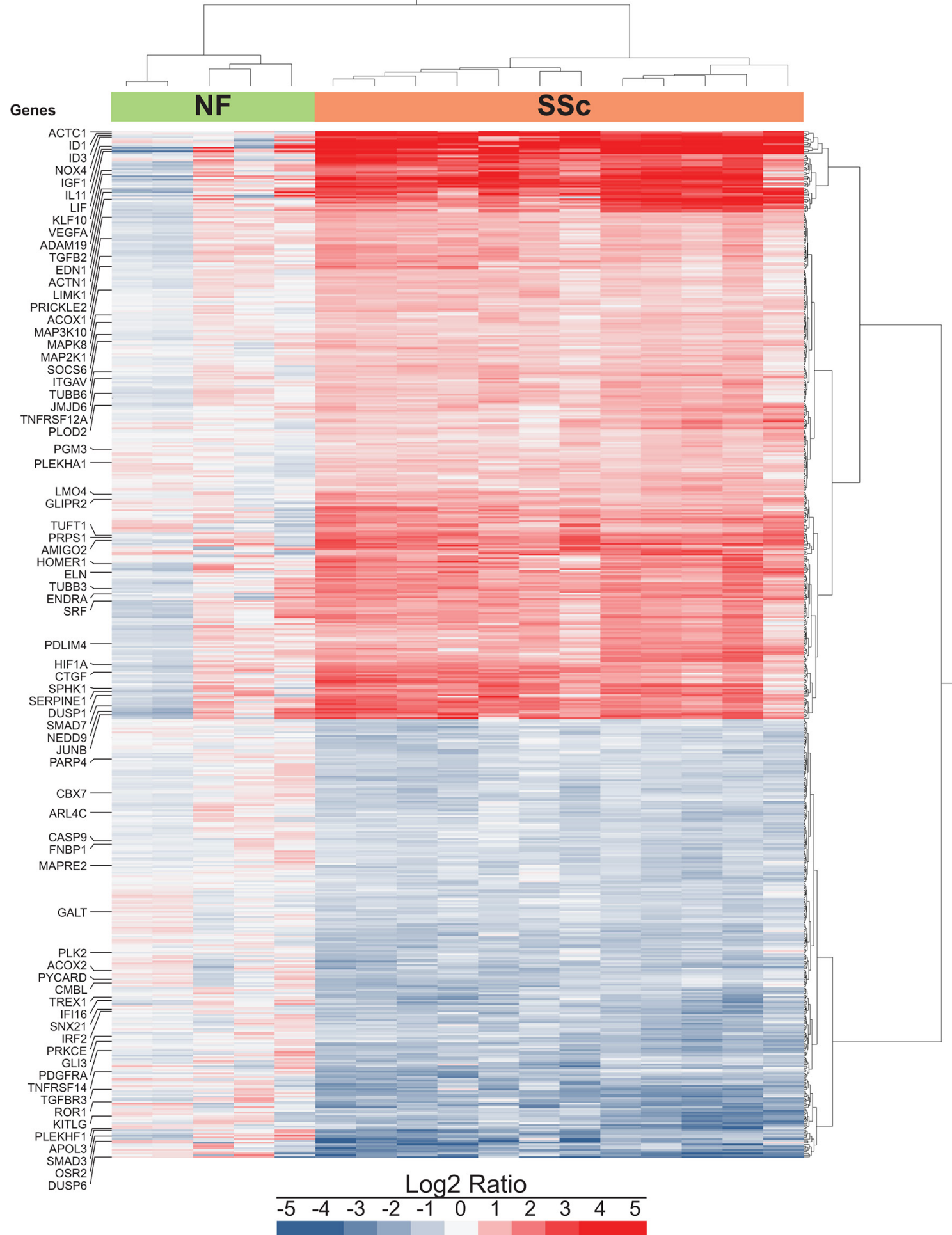

Figure 2 Genes differentially expressed by SSc dermal fibroblasts collected at baseline compared with normal dermal fibroblasts represent a typical profibrotic signature dominated by genes regulated by TGF $\beta$. Gene expression of normal, SSC PBO and SSC TCZ dermal fibroblasts collected at baseline and normalised to the average NF expression. Protein coding RNA showing a median RPKM $\geq 1,1.5$-fold overexpressed or underexpressed compared with $N F$, with an adjusted $p \leq 0.05$, was selected as significantly differentially expressed $(n=578)$. Genes and samples were clustered using the Ward method. BL, baseline; NFs, normal fibroblasts; PBO, placebo; RPKM, reads per kilobase of transcript per million mapped reads; SSc, systemic sclerosis; TCZ, tocilizumab; TGF $\beta$, transforming growth factor beta.

associated with cellular activation. The two pathways grouped in the hormones node are thought to contribute to the pathobiology of SSc, but the exact mechanisms by which this occurs remain ill defined. The inflammation node includes two pathways related to the innate immune system. Three of the four pathways included in the morphogenesis node belong to the bone morphogenetic protein family, and the fourth pathway in this node is related to neuregulin biology. Overall, for the baseline SSc explant dermal fibroblasts (SSc vs NF comparison), the functions and activation status of the top 50 selected pathways depict a highly activated 

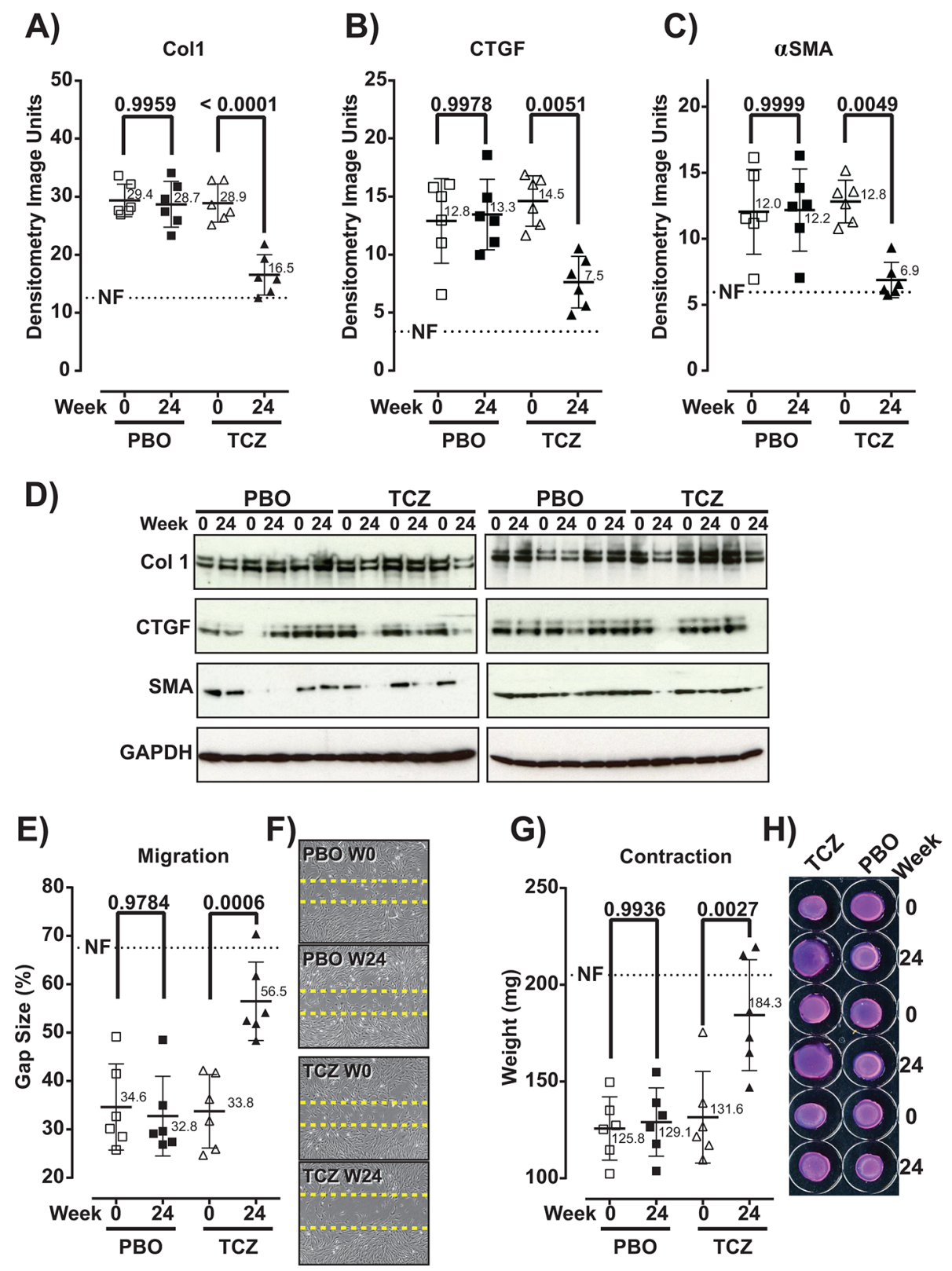

Figure 3 Hallmark functional properties of SSc explant dermal fibroblasts are attenuated after treatment of patients with TCZ for 24 weeks. Comparison of functional properties of explant dermal fibroblasts from PBO-treated and TCZ-treated patients collected at baseline (week 0) and at week 24. Production of Col1 (A), CTGF (B) and $\alpha$ SMA (C); migration activity (E); and contractility (G) were analysed by western blot densitometry of cell lysates $(A-C)$, scratch assay: percentage of remaining gap size $(E)$ and $3 D$ gel contraction assay: weight of the lattice plug (G), respectively. Representative western blot images are presented for each protein analysed (D, lower panel). Western blot of the reference protein GAPDH is shown for the corresponding samples. Representative images of scratch assay performed over an incubation period of 48 hours are shown (F). Representative images of gel contraction assay performed over an incubation period of 24 hours are shown $(\mathrm{H})$. For each graph, the average measure of NFs is depicted as a dotted line. BL, baseline; Col1, collagen alpha 1; CTGF, connective tissue growth factor; NFs, normal fibroblasts; PBO, placebo; $\alpha$ SMA, alpha-smooth muscle actin; SSc, systemic sclerosis; TCZ, tocilizumab.

fibrotic, motile and contractile phenotype dominated by TGF $\beta$ superfamily member-regulated biology, where positive regulators $(n=47)$ are activated and negative regulators $(n=3)$ are predicted to be inhibited. In contrast, for the dermal fibroblasts collected from patients treated with TCZ for 24 weeks (TCZ week 24 vs baseline comparison), the same 50 pathways are predicted to have the opposite activation status. Notably, the level of inhibition observed with TCZ generally matches the levels of activation observed at baseline. Thus, in keeping with the functional assay and the gene expression data, the pathway analysis results indicate that the 24-week treatment of patients with TCZ almost completely reverses the SSc-associated fibrotic phenotype of explant dermal fibroblasts.

\section{DISCUSSION}

This study provides exceptional insight into the molecular pathology of dermal fibroblasts in severe SSc and unique insight into the likely key pathways and mediators involved in SSc pathogenesis, building on the original observations of LeRoy. ${ }^{28}$ By linking the ex vivo data to promising clinical responses in a PBO-controlled trial of TCZ, our results also elucidate new and 


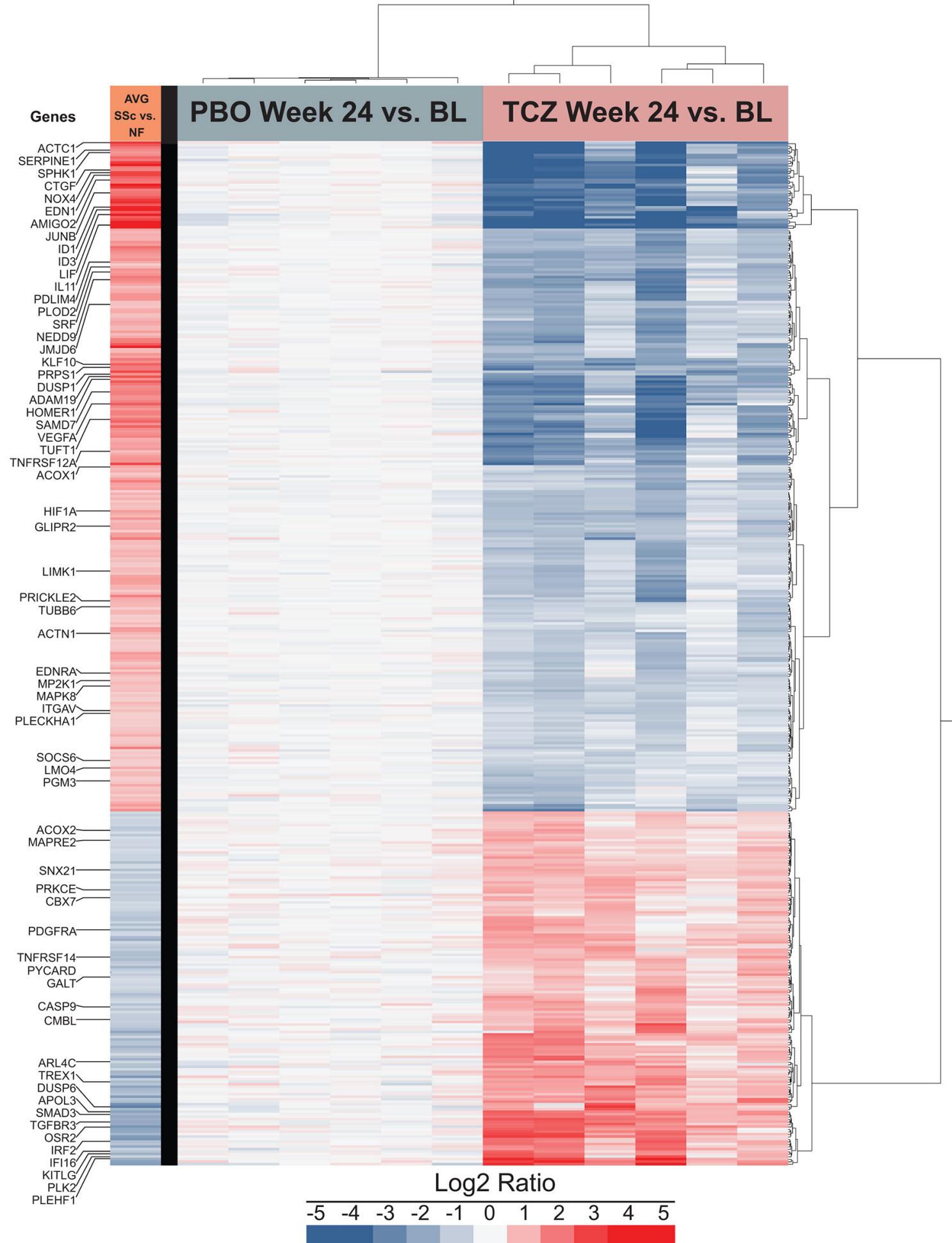

Figure 4 The profibrotic expression profile of SSC dermal fibroblasts tends to be normalised by 24-week treatment of patients with TCZ and tends to be stable in PBO-treated patients. Gene expression levels of SSC PBO and SSC TCZ dermal fibroblasts were measured at week 24 and normalised to matching baseline expression levels. Protein coding RNA showing a median RPKM $\geq 1,1.5$-fold overexpressed or underexpressed at week 24 compared with baseline with an adjusted $p \leq 0.05$ was marked as significantly differentially expressed $(n=2136)$. For clarity, only the subset of 411 genes differentially expressed in both SSc versus NF and in TCZ week 24 versus baseline is shown. Genes and samples were clustered using the Ward method. The first column represents the average differential expression of SSc baseline samples compared with NF samples. AVG, average; BL, baseline; NF, normal fibroblast; PBO, placebo; RPKM, reads per kilobase of transcript per million mapped reads; SSc, systemic sclerosis; TCZ, tocilizumab.

important mechanisms by which TCZ may exert an antifibrotic effect and build on data from whole-skin gene expression analysis from the faSScinate phase 2 study. ${ }^{21}$ Although it was demonstrated that treatment with TCZ for 24 weeks resulted in a specific downregulation of genes associated with $\mathrm{M} 2$ macrophages and with the IL-6 pathway, little specific effect of TCZ treatment could be 


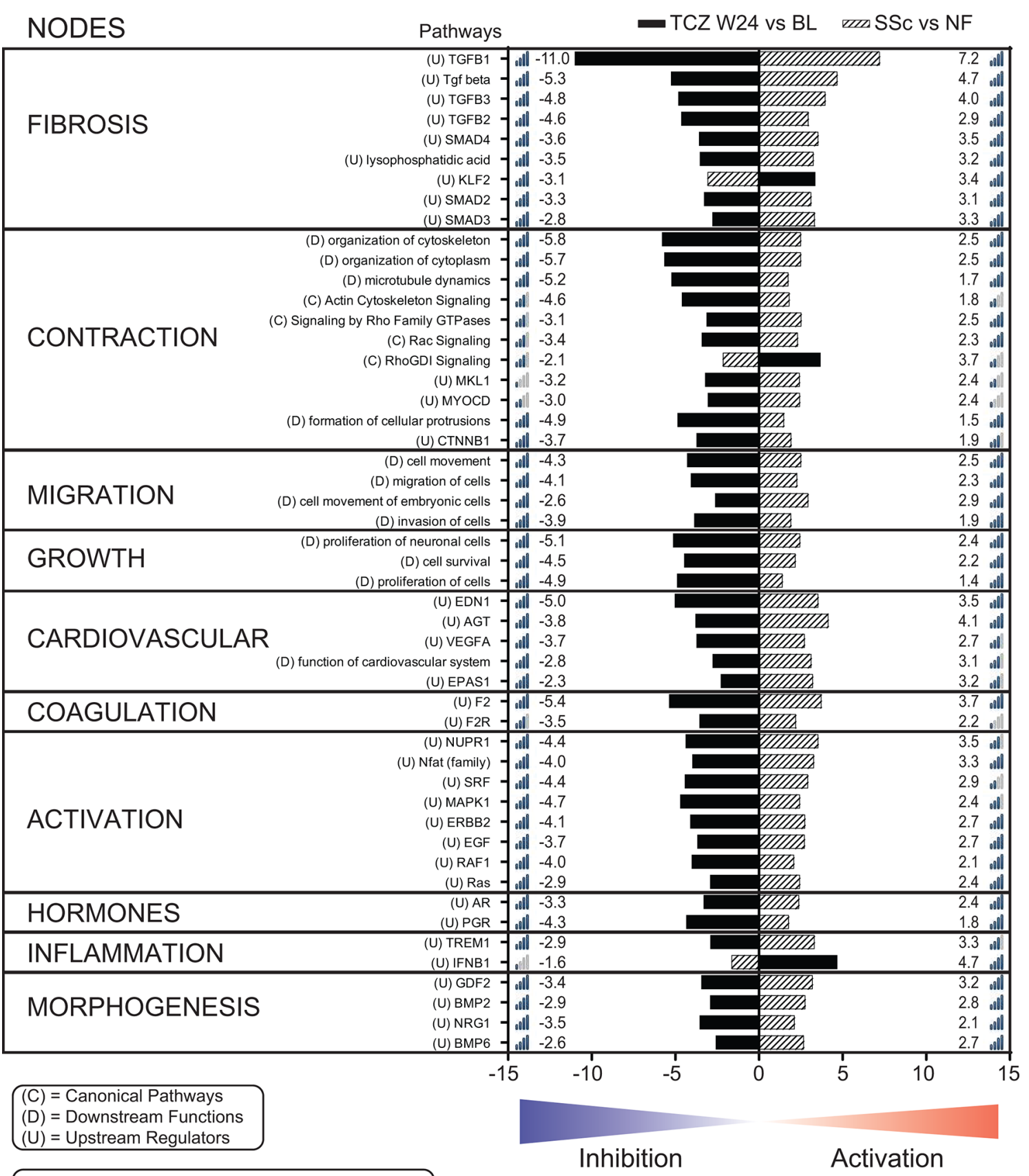

\begin{tabular}{|ccccc|}
\hline & $<0.05$ & $<0.01$ & $<0.001$ & $<0.0001$ \\
\hline
\end{tabular}

\section{Z Scores}

Figure 5 Treatment with TCZ for 24 weeks normalises the activation status of molecular and functional pathways associated with the biology of SSc fibroblasts. Activation status Z scores for the top 50 associated pathways grouped according to functional nodes. Genes significantly differentially expressed in the SSC versus NF (hatched bars) and the TCZ W24 versus baseline (solid black bars) comparisons (online supplementary figure S1) were analysed with IPA (Qiagen). Differential gene expression data for all differentially expressed genes are available in online supplementary appendix S2. BL, baseline; EGF, epidermal growth factor; IPA, Ingenuity Pathway Analysis; MAPK1, mitogen-activated protein kinase 1; NF, normal fibroblast; SSc, systemic sclerosis; TCZ, tocilizumab; TGFß1, tumour necrosis factor beta 1; VEGFA, vascular endothelial growth factor A; W24, week 24.

detected on genes associated with fibrosis at the whole-skin biopsy level. Considering the multicellular complexity and relative heterogeneity of whole-skin biopsy samples used in this study, we hypothesised that the effect of TCZ on fibroblasts might be masked by the gene expression of other cellular compartments. The clinical homogeneity of the faSScinate patients and the access to samples collected from the same patients before and after 24 weeks of treatment with TCZ or PBO allowed us to use relatively small numbers of samples to generate robust and reliable results. As a result, we are able to confirm and expand on the description of the profibrotic properties of SSc dermal fibroblasts at the functional and genomic levels, establish that the profibrotic phenotype of SSc dermal fibroblasts is stable over $\geq 24$ weeks in patients treated with PBO in the absence of immunosuppressant (cyclophosphamide, mycophenolate mofetil or methotrexate) and demonstrate that treatment of SSc patients with TCZ for 24 weeks profoundly alters the biological characteristics of dermal fibroblasts.

The baseline functional and genomic properties of the dermal explant fibroblasts analysed in this study closely match those described in previous publications. ${ }^{232429}$ Specifically, the baseline 


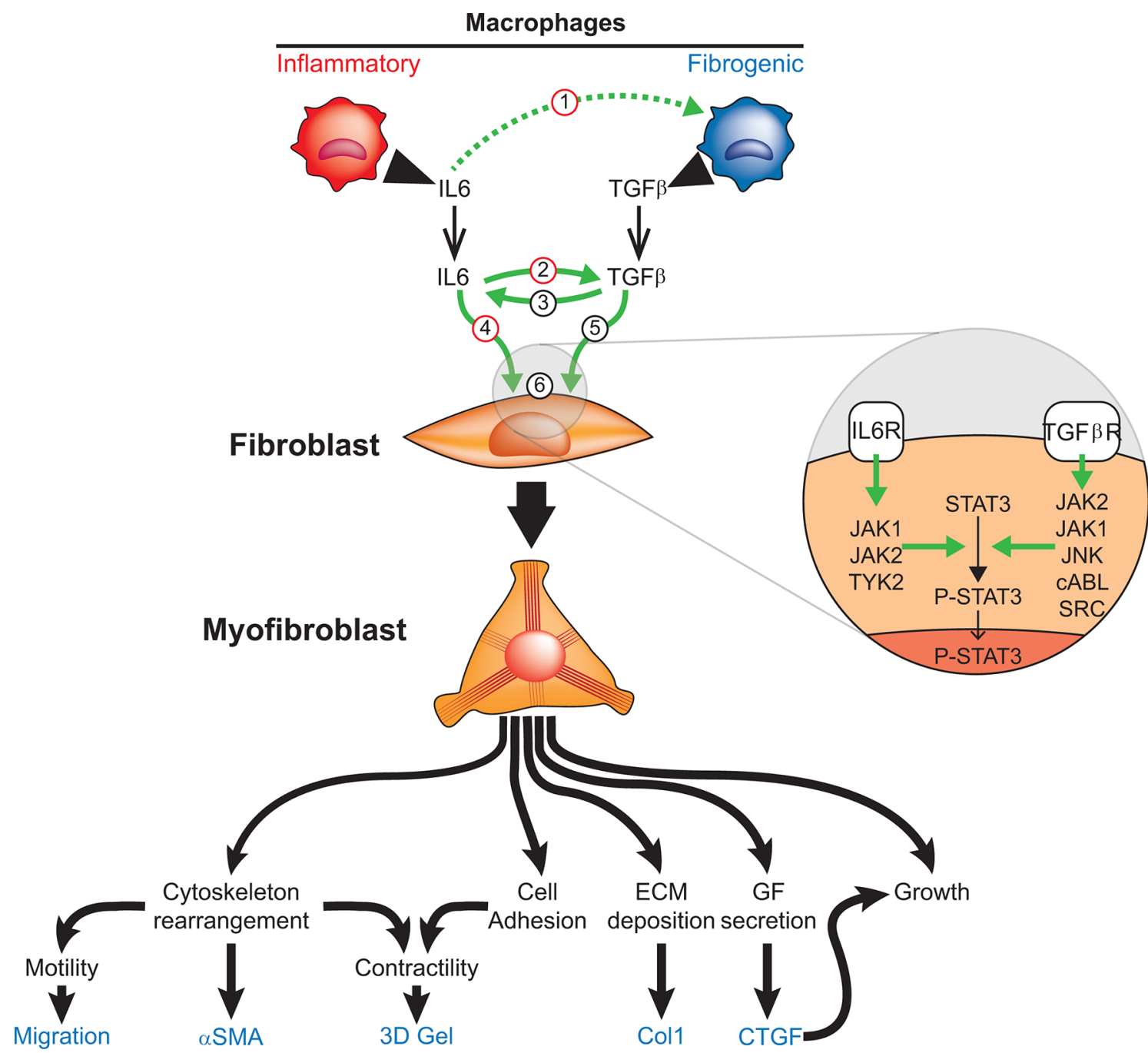

Figure 6 Potential mechanisms of interaction between IL-6 and TGF $\beta$ leading to fibrosis in SSc. (1) Inflammatory macrophage-produced IL-6 enhances fibrogenic macrophage function (potential TCZ target). (2) IL-6 induces TGF $\beta$ production and enhances its signalling in fibroblasts (potential TCZ target). (3) TGF $\beta$ enhances IL-6 production by fibroblasts. (4) IL-6 directly activates fibroblasts to produce profibrotic proteins such as Col1 (potential TCZ target). (5) TGF $\beta$ directly induces fibroblast differentiation into myofibroblasts. (6) Intracellularly, IL-6 classic signalling and TGF $\beta$ alternative signalling converge towards the activation of STAT3 (STAT3 $\rightarrow$ P-STAT3) resulting in aberrant expression of STAT3-dependent genes. aSMA, alpha-smooth muscle actin; Col1, collagen alpha 1; CTGF, connective tissue growth factor; ECM, extracellular matrix; GF, growth factor; IL-6, interleukin-6; JAK, Janus-activated kinase; JNK, Jun N-terminal kinase; P-STAT, phosphorylated STAT; STAT, signal transducer and activator of transcription; TGF $\beta$, transforming growth factor beta; TGF $\beta R$, transforming growth factor beta receptor; TYK, tyrosine kinase.

SSc dermal fibroblasts produced elevated levels of profibrotic proteins and of migration and contractility activities and differentially expressed a distinct set of fibrosis-related genes with a high prevalence of TGF $\beta$-regulated genes. Considering that the faSScinate patients were experiencing active progressive disease at study entry, ${ }^{21}$ our observation is consistent with the work of Sargent $e t a l,{ }^{24}$ who reported that the differential expression of genes regulated by TGF $\beta$ is associated with more severe disease when detected at the whole-biopsy level. Over the years, the study of explant dermal fibroblasts in vitro has provided valuable insight into the pathobiology of SSc. ${ }^{6722283031}$ The fact that the phenotypic abnormalities of SSc dermal fibroblasts persist in vitro over multiple passages has been critical to this line of investigation. However, it is important to note that dermal fibroblasts are heterogeneous and that they manifest different properties as a function of their microenvironment. ${ }^{32}$ The potential selection pressure imposed by isolation, plating and passage procedures makes it likely that only a subset of fibroblasts is accessible to in vitro studies. These may represent fibroblasts that are more motile, better able to acclimate to the culture conditions or have higher growth potential, thus perhaps representing stem cell population(s). For any of these reasons, we might have selected a fibroblast subpopulation in the explant culture conditions more sensitive to the effects of IL-6 inhibition.

We used IPA (Qiagen) to associate gene expression with biological pathways, focusing on the molecular and cellular biology of relevance to fibroblasts. The top 50 pathways associated with SSc differentially expressed genes could be grouped into 10 core biological nodes that covered two levels of biology: biology intrinsic to fibroblasts (fibrosis, contraction, migration, growth and activation) and biology extrinsic to fibroblasts (cardiovascular, coagulation, hormones, inflammation and morphogenesis). The pathways forming the intrinsic group reflect canonical features of fibrotic fibroblasts ${ }^{5}$ with a dominant contribution by TGF $\beta$. This dominance of TGF $\beta$-related biology is apparent in the fibrosis node and in the contraction node, where activation of Rho GTPases can be triggered by TGF $\beta$ in a SMAD-independent process, ${ }^{33}$ and in the activation node, where Ras, RAF1 and 
ERK are part of one branch of SMAD-independent TGF $\beta$ signalling. ${ }^{34}$ Conversely, the pathways forming the extrinsic group represent biological processes that link inflammation and vasculopathy to fibrosis. For example, not only are the cardiovascular pathways EDN1 and AGT potent mediators of vasoconstriction, ${ }^{35}$ they are profibrotic mediators relevant to the pathobiology of SSc. ${ }^{37} 38$ This is notable considering the prominent vasculopathy of SSc and the putative benefit for patients with SSc of drugs that target these pathways. Similarly, in addition to playing a key role in response to angiogenesis, vascular endothelial growth factor A (VEGFA) and the VEGFA-inducing transcription factor endothelial PAS domain-containing protein 1 amplify fibrosis, ${ }^{39}$ creating a link between response to hypoxia and fibrosis. Although excessive inhibition of angiogenesis could be detrimental, our data support normalisation of the activation status of cardiovascular-related pathways rather than complete blockade of these pathways, which might be detrimental for wound healing or tissue repair.

Patient treatment with TCZ for 24 weeks profoundly affected the properties of explant dermal fibroblasts. It is not possible to determine whether treatment with TCZ modified the phenotype of resident cells or the cellular composition of the dermis; both these mechanisms are non-exclusive. Independent of the exact mechanism, the effect of IL-6R $\alpha$ blockade on biological pathways dominated by TGF $\beta$-related biology is striking and highlights the interaction between IL- 6 and TGF $\beta$. Although TGF $\beta$ is the quintessential profibrotic factor, ${ }^{9}$ IL- 6 has also been shown to induce the production of fibrotic markers (CTGF, $\alpha$ SMA and collagen 1) by human fibroblasts in vitro. ${ }^{15}$ Hence, IL-6 and TGF $\beta$ may form a self-sustaining loop leading to fibrosis that could be interrupted by blockade of either or both cytokines. This is supported by recent work demonstrating that STAT3, the main downstream signalling element for IL-6, is an important TGF $\beta$-dependent molecular checkpoint of fibrosis in SSc. ${ }^{40}$ Altogether, these results and our observations at the whole-biopsy level ${ }^{21}$ suggest a potential interaction network between IL- 6 and TGF $\beta$ involving fibroblasts and macrophages that leads to fibrosis in SSc (figure 6). Thus, blocking IL-6 signalling with TCZ may represent a novel approach to control both the TGF $\beta$ pathway and the inflammatory pathways, supporting further exploration of IL-6 and related mediators as potential therapeutic targets in SSc and other inflammation-driven fibrotic diseases.

A unique strength of this study is longitudinal sampling of well-characterised and otherwise untreated SSc patients with clinically active disease receiving TCZ or PBO treatment. The combination of clinical homogeneity of patients, repeat sampling at two time points and quality of characterisation assays allowed us to use relatively small numbers of samples to generate robust and reliable results. In addition, the combination of well-characterised functional assays with gene expression analysis using RNA-Seq provides a rare window into the biology of active SSc. However, this study does not address the apparent discrepancy between biological and clinical effects of TCZ on skin fibrosis. Although additional studies are needed to fill this gap, we hypothesise that TCZ primarily affects de novo fibrosis and has little impact on the reduction of established fibrosis. Hence, in established skin fibrosis, the clinical effect of TCZ would be substantially delayed after its biological effect.

In conclusion, this study in the field of SSc translational research sheds new light on SSc biology by leveraging the conduct of a well-designed clinical trial in SSc and rigorous functional and molecular analysis of skin biopsy samples to elucidate the links between IL-6 biology and other key pathways and mediators in determining the hallmark profibrotic phenotype of
SSc dermal fibroblasts. Moreover, our findings strongly support further exploration of IL- 6 and related mediators as potential therapeutic targets in SSc and other fibrotic diseases.

Acknowledgements The authors would like to thank all the investigators and the faSscinate study team.

Contributors All authors were involved in drafting the manuscript or revising it critically for important intellectual content, made substantial contributions to the conception or design of the study or the acquisition, analysis or interpretation of data, approved the final version of the manuscript to be published and agree to be accountable for all aspects of the work in ensuring that questions related to the accuracy or integrity of the work are appropriately investigated and resolved.

Funding This study was funded by F Hoffmann-La Roche. Writing and editorial assistance was provided by Maxwell Chang, BSc, and Sara Duggan, PhD, on behalf of F Hoffmann-La Roche Ltd. Additional funding from EULAR via the Orphan Diseases Programme is gratefully acknowledged.

Competing interests CPD reports personal fees from Roche/Genentech, Actelion, GlaxoSmithKline, Sanofi-Aventis, Inventiva and Boehringer-Ingelheim; grants and personal fees from Bayer; and grants from CSL Behring during the conduct of the study. VHO and SX have nothing to disclose. HC-H is an employee of Genentech. ZM, JS and TS are employees of and own stock in Genentech. RL reports personal fees from Biocon and Merck, grants from Elpidera and grants and personal fees from PRISM Biolab outside of the submitted work. DK reports personal fees from Actelion, Cytori, EMD Serono, Gilead, GlaxoSmithKline, SanofiAventis, Corbus, ChemomAb, Eicos and UCB Pharma; grants from Bristol-Myers Squibb, the National Institutes of Health and Pfizer; grants and personal fees from Bayer, Roche/Genentech and Boehringer-Ingelheim during the conduct of the study; and personal fees from AstraZeneca outside the submitted work. AJ is an employee of Genentech, owns stock and stock options in Roche and has a patent issued for tocilizumab.

\section{Patient consent Obtained.}

Ethics approval The study protocol was approved by the institutional review boards or ethics committees before the study commenced. All subjects provided written consent. The study was conducted in accordance with the Declaration of Helsinki and with Good Clinical Practice. 'Control' skin biopsy samples were obtained with informed consent from healthy volunteers (London-Hampstead NRES Committee, MREC Reference 6398)

Provenance and peer review Not commissioned; externally peer reviewed.

Open access This is an open access article distributed in accordance with the Creative Commons Attribution Non Commercial (CC BY-NC 4.0) license, which permits others to distribute, remix, adapt, build upon this work non-commercially, and license their derivative works on different terms, provided the original work is properly cited and the use is non-commercial. See: http://creativecommons.org/ licenses/by-nc/4.0/

(c) Article author(s) (or their employer(s) unless otherwise stated in the text of the article) 2018. All rights reserved. No commercial use is permitted unless otherwise expressly granted.

\section{REFERENCES}

1 Denton CP, Black CM, Abraham DJ. Mechanisms and consequences of fibrosis in systemic sclerosis. Nat Clin Pract Rheumatol 2006;2:134-44.

2 Eckes B, Zigrino P, Kessler D, et al. Fibroblast-matrix interactions in wound healing and fibrosis. Matrix Biol 2000;19:325-32.

3 Denton CP, Hughes M, Gak N, et al. BSR and BHPR guideline for the treatment of systemic sclerosis. Rheumatology 2016;55:1906-10.

4 Shand L, Lunt M, Nihtyanova S, et al. Relationship between change in skin score and disease outcome in diffuse cutaneous systemic sclerosis: application of a latent linear trajectory model. Arthritis Rheum 2007;56:2422-31.

5 Gilbane AJ, Denton CP, Holmes AM. Scleroderma pathogenesis: a pivotal role for fibroblasts as effector cells. Arthritis Res Ther 2013;15:215.

6 Denton CP, Xu S, Welsh Kl, et al. Scleroderma fibroblast phenotype is modulated by endothelial cell co-culture. J Rheumatol 1996;23:633-8.

7 Denton CP, Shi-Wen X, Sutton A, et al. Scleroderma fibroblasts promote migration of mononuclear leucocytes across endothelial cell monolayers. Clin Exp Immunol 1998; 114:293-300.

8 Sato S. Abnormalities of adhesion molecules and chemokines in scleroderma. Curr Opin Rheumatol 1999;11:503-7.

9 Denton CP, Abraham DJ. Transforming growth factor-beta and connective tissue growth factor: key cytokines in scleroderma pathogenesis. Curr Opin Rheumatol 2001;13:505-11.

10 Varga J, Whitfield ML. Transforming growth factor-beta in systemic sclerosis scleroderma. Frontiers in Bioscience 2009:51:226-35.

11 O'Reilly S, Cant R, Ciechomska M, et al. Interleukin-6: a new therapeutic target in systemic sclerosis? Clin Trans/ Immunology 2013;2:e4. 
12 Kadono T, Kikuchi K, Ihn H, et al. Increased production of interleukin 6 and interleukin 8 in scleroderma fibroblasts. J Rheumato/ 1998:25:S191-301.

13 Desallais L, Avouac J, Fréchet M, et al. Targeting IL- 6 by both passive or active immunization strategies prevents bleomycin-induced skin fibrosis. Arthritis Res Ther 2014; 16:R157.

14 De Lauretis A, Sestini P, Pantelidis P, et al. Serum interleukin 6 is predictive of early functional decline and mortality in interstitial lung disease associated with systemic sclerosis. J Rheumatol 2013:40:435-46.

15 Khan K, Xu S, Nihtyanova S, et al. Clinical and pathological significance of interleukin 6 overexpression in systemic sclerosis. Ann Rheum Dis 2012;71:1235-42.

16 Ma F, Li Y, Jia L, et al. Macrophage-stimulated cardiac fibroblast production of IL-6 is essential for TGF $\beta /$ Smad activation and cardiac fibrosis induced by angiotensin II. PLoS One 2012;7:e35144.

17 O'Reilly S, Ciechomska M, Cant R, et al. Interleukin-6 (IL-6) trans signaling drives a STAT3-dependent pathway that leads to hyperactive transforming growth factor- $\beta$ (TGF- $\beta$ ) signaling promoting SMAD3 activation and fibrosis via Gremlin protein. J Biol Chem 2014:289:9952-60.

18 Wang JH, Zhao L, Pan X, et al. Hypoxia-stimulated cardiac fibroblast production of IL- 6 promotes myocardial fibrosis via the TGF- $\beta 1$ signaling pathway. Lab Invest 2016;96:839-52.

19 Elias JA, Lentz V, Cummings PJ. Transforming growth factor-beta regulation of IL-6 production by unstimulated and IL-1-stimulated human fibroblasts. J Immunol 1991; 146:3437-43.

20 Michaeloudes C, Sukkar MB, Khorasani NM, et al. TGF- $\beta$ regulates Nox4, MnSOD and catalase expression, and IL-6 release in airway smooth muscle cells. Am J Physiol Lung Cell Mol Physiol 2011;300:L295-L304.

21 Khanna D, Denton CP, Jahreis A, et al. Safety and efficacy of subcutaneous tocilizumab in adults with systemic sclerosis (faSScinate): a phase 2, randomised, controlled trial. Lancet 2016;387:2630-40

22 Xu SW, Liu S, Eastwood M, et al. Rac inhibition reverses the phenotype of fibrotic fibroblasts. PLoS One 2009;4:e7438.

23 Shi-wen X, Eastwood M, Stratton RJ, et al. Rosiglitazone alleviates the persistent fibrotic phenotype of lesional skin scleroderma fibroblasts. Rheumatology 2010;49:259-63.

24 Sargent JL, Milano A, Bhattacharyya S, et al. A TGFbeta-responsive gene signature is associated with a subset of diffuse scleroderma with increased disease severity. J Invest Dermatol 2010:130:694-705.

25 Tan AB, Kress S, Castro L, et al. Cellular re- and de-programming by microenvironmental memory: why short TGF- $\beta 1$ pulses can have long effects. Fibrogenesis Tissue Repair 2013;6:12.
26 Renzoni EA, Abraham DJ, Howat $\mathrm{S}$, et al. Gene expression profiling reveals novel TGFbeta targets in adult lung fibroblasts. Respir Res 2004;5:24.

27 Tourkina E, Gooz P, Oates JC, et al. Curcumin-induced apoptosis in scleroderma lung fibroblasts: role of protein kinase cepsilon. Am J Respir Cell Mol Biol 2004;31:28-35.

28 Leroy EC. Connective tissue synthesis by scleroderma skin fibroblasts in cell culture. J Exp Med 1972;135:1351-62.

29 Hinchcliff $M$, Huang CC, Ishida W, et al. Imatinib mesylate causes genome-wide transcriptional changes in systemic sclerosis fibroblasts in vitro. Clin Exp Rheumatol 2012:30(2 Suppl 71):S86-96.

30 Trojanowska M. What did we learn by studying scleroderma fibroblasts? Clin Exp Rheumatol 2004:22(3 Suppl 33):S59-S63.

31 Johnson ME, Mahoney JM, Taroni J, et al. Experimentally-derived fibroblast gene signatures identify molecular pathways associated with distinct subsets of systemic sclerosis patients in three independent cohorts. PLoS One 2015;10:e0114017.

32 Sriram G, Bigliardi PL, Bigliardi-Qi M. Fibroblast heterogeneity and its implications for engineering organotypic skin models in vitro. Eur J Cell Biol 2015;94:483-512.

33 Edlund $\mathrm{S}$, Landström $\mathrm{M}$, Heldin $\mathrm{CH}$, et al. Transforming growth factor-beta-induced mobilization of actin cytoskeleton requires signaling by small GTPases Cdc42 and RhoA. Mol Biol Cell 2002;13:902-14.

34 Derynck R, Zhang YE. Smad-dependent and Smad-independent pathways in TGF-beta family signalling. Nature 2003;425:577-84.

35 Davenport AP, Hyndman KA, Dhaun N, et al. Endothelin. Pharmacol Rev 2016:68:357-418

36 Cockcroft JR, O'Kane KP, Webb DJ. Tissue angiotensin generation and regulation of vascular tone. Pharmacol Ther 1995:65:193-213.

37 Shi-Wen X, Chen Y, Denton CP, et al. Endothelin-1 promotes myofibroblast induction through the ETA receptor via a rac/phosphoinositide 3-kinase/Akt-dependent pathway and is essential for the enhanced contractile phenotype of fibrotic fibroblasts. Mol Biol Cell 2004:15:2707-19.

38 Kawaguchi Y, Takagi K, Hara M, et al. Angiotensin II in the lesional skin of systemic sclerosis patients contributes to tissue fibrosis via angiotensin II type 1 receptors. Arthritis Rheum 2004;50:216-26.

39 Maurer B, Distler A, Suliman YA, et al. Vascular endothelial growth factor aggravates fibrosis and vasculopathy in experimental models of systemic sclerosis. Ann Rheum Dis 2014:73:1880-7.

40 Chakraborty D, Šumová B, Mallano T, et al. Activation of STAT3 integrates common profibrotic pathways to promote fibroblast activation and tissue fibrosis. Nat Commun 2017:8:1130. 